\title{
The effect of the combination of reducing and oxidising agents on the viscoelastic properties of dough and sensory characteristics of buns
}

\author{
Pavlína Pečivová, Vladimír Pavlinek and Jan Hrabě Abstract
}

\begin{abstract}
BACKGROUND: The effects of mixtures of reducing and oxidising agents on the rheological characteristics and quality of wheat flour dough and the sensory characteristics of buns were studied.

RESULTS: In chemical analyses, no differences between control dough and doughs with individual mixtures of additives were found. Rheological measurements showed that increasing addition of L-ascorbic acid + L-tryptophan mixture to the dough increased its tenacity and decreased its extensibility. Increasing addition of L-ascorbic acid + L-threonine mixture also led to an increase in dough tenacity but to a slight increase in dough extensibility. Increasing addition of L-ascorbic acid + inactivated dry yeast mixture to the dough resulted in a decrease in tenacity and an increase in extensibility. Two other additive mixtures, L-ascorbic acid + L-cysteine hydrochloride monohydrate and L-cysteine p.a. + inactivated dry yeast, reduced both the tenacity and extensibility of the dough. In sensory analyses, differences in dryness, pliability, sensation when swallowing, quality and texture of buns with additive mixtures were found in comparison with control buns, but gumminess did not change significantly with increasing amount of additive mixtures.
\end{abstract}

Keywords: gluten; rheology; sensory analysis; additive

\section{INTRODUCTION}

Among cereal flours, only wheat flour can form three-dimensional viscoelastic dough when mixed with water. Characterisation of the rheological properties of dough is effective in predicting its processing behaviour and controlling the quality of food products. ${ }^{1}$

Wheat proteins, especially gluten protein and its fractions, are generally recognised as the most important factors governing the viscoelastic properties of flour dough and its breadmaking quality. ${ }^{2-4}$ High protein content is often related to good breadmaking quality. However, protein quantity alone cannot explain all variations in breadmaking quality. Other factors such as protein quality are also important. ${ }^{5}$ Wheat quality is determined by high-molecular-weight glutenin subunits (HMW-GS) ${ }^{6}$ and the molecular weight distribution (MWD) of wheat glutenin. ${ }^{7}$ The MWD is determined by the ratios of monomeric/polymeric proteins, HMW-GS/low-molecular-weight glutenin subunits (LMW-GS) and glutenin/gliadin. ${ }^{5,7-12}$ Indeed, the unusual properties of gluten proteins enable wheat flour to be transformed into dough with suitable properties for breadmaking. These properties are unique and cannot even be closely reproduced in dough made from other cereals. ${ }^{13}$

Traditionally, gluten proteins are divided into two roughly equal fractions according to their solubility in alcohol/water solutions of gluten (e.g. $600 \mathrm{~mL} \mathrm{~L} \sim^{1}$ ethanol), namely soluble gliadins and insoluble glutenins. Gliadins are monomeric proteins with a molecular weight of $30-80 \mathrm{kDa}$ and can be further classified into four groups ( $a, p, y$ and it»). ${ }^{1,14}$

The glutenin fraction comprises aggregated proteins linked by interchain disulfide bonds, whose sizes range from about $5 \times 10^{5}$ to more than $1 \times 10^{7} .{ }^{15,16}$ Glutenins are polymeric proteins composed of LMW-GS and HMW-GS of 12-60 and 60-120 kDa respectively ${ }^{1,14}$ and are among the largest proteins. The actual sizes of the largest glutenin polymers have not yet been accurately determined because of their enormity. ${ }^{17}$ Another reason for difficulties in the size determination of glutenins is their poor solubility in conventional buffers. ${ }^{13}$ However, a significant part of glutenin cannot be solubilised without changing its structure. This high insolubility of glutenin explains why, despite considerable efforts over more than a century, relatively few details on the structure 
of glutenin are available. What is known, however, is that glutenin is built of glutenin subunits (GS) that are linked via disulfide bonds. These GS can be liberated by reduction or oxidation of the disulfide bonds with reducing or oxidising agents. ${ }^{13}$

As stated above, the functionality of gluten in wheat flour used for breadmaking is strongly determined by the MWD of glutenin, the occurrence of covalent and non-covalent bonds between glutenin molecules and the interactions between glutenin and other flour constituents. The most prominent linkages are disulfide bonds, as they hold together the GS. Hence oxidising and reducing agents that have a strong impact on the dough thiol/disulfide system can affect the polymerisation of GS and thereby change the mechanical and rheological properties of dough. ${ }^{13,18}$ Even very low concentrations of endogenous glutathione drastically weaken dough and increase its extensibility through thiol-disulphide interchange. ${ }^{13}$ Reducing agents such as L-cysteine may be added to weaken the dough structure. LCysteine can also be used in combination with oxidants. During kneading, cysteine cleaves disulfide bonds, which facilitates the distribution of gluten protein in dough and improves dough extensibility. ${ }^{13}$ Cysteine addition increases the fluid-like properties of dough: dough hardness, resistance to extension and storage modulus $\left(G^{\prime}\right)$ are lower while adhesiveness and extensibility are higher when cysteine is added. ${ }^{19,20}$

Cysteine reduces disulfide bonds between proteins and weakens the gluten network of dough, thereby decreasing the elastic (solidlike) component of the dough, aiding in dough relaxation and reducing mixing time. ${ }^{21}$

Generally, cysteine addition leads to an increase in water absorption, a reduction in dough development time and a decrease in dough stability. Addition of L-cysteine hydrochloride (1 $\mathrm{mLLr}^{1}$ ) increases water absorption but decreases dough development time and dough stability; high levels of cysteine addition increase the compressibility, indicating a decrease in crumb softness, whereas low levels of cysteine addition decrease the compressibility, indicating a softer crumb. ${ }^{21}$

Ascorbic acid is widely used as a flour improver in bread products. ${ }^{22}$ Although itself a reducing agent, ascorbic acid can exert an oxidising effect on dough properties after its oxidation by atmospheric oxygen during kneading and maturing. It is now generally accepted that the actual improving compound is its first relatively stable oxidation product, i.e. dehydroascorbic acid. The oxidation of ascorbic acid to dehydroascorbic acid takes place quickly and is already completed before the end of the mixing stage. ${ }^{22-24}$ It is able to create bonds and to strengthen the gluten network in dough.

Amino acids such as L-threonine and L-tryptophan are essential amino acids, a minority amount of which are present in wheat flour. Further addition of these amino acids to flour can increase the nutritional value of final bakery products. So far, these amino acids or their mixtures with ascorbic acid have not been added to dough in order to improve its rheological properties and enhance the organoleptic and nutritional qualities of final bakery products. Their effect on the gluten structure of dough and the organoleptic and nutritional qualities of bakery products is almost unknown.

The rheological properties of wheat flour dough are critical for the successful manufacturing of bakery products, because they determine the dough behaviour during mechanical handling and thus affect the quality of the final product. ${ }^{25}$ Much of the research in bakery processing has been devoted to the testing of fresh dough. ${ }^{26,27}$

In wheat breadmaking, flour, water, salt, yeast and/or other micro-organisms are mixed into viscoelastic dough. During dough mixing, gluten proteins are transformed into a continuous cohesive viscoelastic gluten protein network. ${ }^{13,28}$ During dough fermentation, further changes in the gluten protein network are evident from the observation that gluten proteins become less extractable again. ${ }^{29,30}$ During dough fermenting, the gluten protein network plays a major role in retaining the carbon dioxide produced during fermentation and during the initial stages of baking. Gas retention properties in turn determine the loaf volume and crumb structure of the resulting bread. $^{13}$

During baking, dramatic changes occur in gluten proteins. These are probably a combination of changes in protein surface hydrophobicity, sulfhydryl-disulphide interchanges and formation of new disulfide crosslinks. ${ }^{31-34}$ As a result of these heat-induced changes as well as those of starch, the typical foam structure of baked bread is formed..$^{13}$

The aim of this study was to determine the influence of individual combinations of reducing and oxidising agents on the change in viscoelastic properties of wheat flour dough and also the effect of their addition on modification or improvement in quality of bakery products. Amino acids such as L-threonine and L-tryptophan alone or in combination with other amino acids and L-ascorbic acid are not commonly used to modify quality parameters in the baking industry.

\section{MATERIALS AND METHODS}

\section{Materials}

Wheat flour

For the assessment, common commercial wheat flour T 530 (quantitative parameters: moisture content, $143 \mathrm{~g} \mathrm{~kg}^{-1}$; gluten content in dry matter, $364 \mathrm{~g} \mathrm{~kg}^{-1}$; falling number, $\left.339 \mathrm{~s}\right)$ provided by Penam s.r.o. (Kroměřižz, Czech Republic) was used.

\section{Additives}

Five different combinations of reducing and oxidising agents were selected. The reducing agents used were L-cysteine p.a. (Merck KGaA, Darmstadt, Germany), L-cysteine hydrochloride monohydrate (Ireks $\mathrm{GmbH}$, Eppelborn, Germany), inactivated dry yeast (Ireks $\mathrm{GmbH}$ ) and L-ascorbic acid (Merck KGaA). The oxidising agents used were Lthreonine (Merck KGaA) and L-tryptophan (Merck KGaA).

The first four mixtures comprised wheat flour, L-ascorbic acid (AsA) and a selected additive, either L-tryptophan (Try), L-threonine (Thr), inactivated dry yeast (IDY) or L-cysteine hydrochloride monohydrate (CYS), while the fifth mixture comprised wheat flour, L-cysteine p.a. (Cys) and IDY.

The composition of and amount of additives in individual mixtures are given in Table 1.

\section{Methods}

Chemical analysis

Each sample was characterised by dry matter content and $\mathrm{pH}$. Dry matter content was determined according to ISO 560 116-3

(1995) . Model dough $\mathrm{pH}$ was measured three times using a $\mathrm{pH}$ meter with a glass electrode.

Rheological measurement and characteristics The rheological characteristics of wheat flour dough were measured using a Chopin AlveoConsistograph (Tripette \& Renaud, France) according to ISO 5530-4 (2002) and ICC Method No. 171 


\begin{tabular}{|c|c|c|c|}
\hline \multirow[b]{2}{*}{ Group } & \multirow[b]{2}{*}{ Mixture of additives: } & \multicolumn{2}{|c|}{ Amount of additives in mixture ig kg $\mathrm{g}^{-\mathrm{i}}$} \\
\hline & & AsA & Try \\
\hline \multirow[t]{4}{*}{ I } & $A 5 A+T r y \mid$ & 1.0 & 2.0 \\
\hline & $A s A-T r y l I$ & 1.0 & 3.0 \\
\hline & $A \subseteq A-T r y \mid \| I$ & 1.0 & 4.0 \\
\hline & & AsA & Thr \\
\hline \multirow[t]{4}{*}{ II } & $\mathrm{ASA}_{\mathrm{S}}-\mathrm{Thrl}$ & 1.0 & 2.0 \\
\hline & AsA - Thrll & 1.0 & 3.0 \\
\hline & $A s A-T h r \mid l l$ & 1.0 & 4.0 \\
\hline & & AsA & $\mathrm{IDY}$ \\
\hline \multirow[t]{4}{*}{ III } & $A S A+|D Y|$ & 1.0 & 2.0 \\
\hline & $A_{5} A+|D Y| \mid$ & 1.0 & 3.0 \\
\hline & $A S A+I D Y H \|$ & 1.0 & 4.0 \\
\hline & & ASA & CYS \\
\hline \multirow[t]{4}{*}{$\mathrm{v}$} & $A S A+C Y S r$ & 1.0 & 0.2 \\
\hline & $A S A+C Y S M$ & 1.0 & 0.5 \\
\hline & ASA + CY5III & 1.0 & 0.6 \\
\hline & & Cys & IDY \\
\hline \multirow[t]{3}{*}{ V } & $C y s+|D Y|$ & 0.1 & 1.0 \\
\hline & $C y 5+\left|D Y Y^{\prime}\right|$ & 0.7 & 2.0 \\
\hline & $C y s+\left|D Y^{\prime}\right| \mid I$ & 0.1 & 3.0 \\
\hline \multicolumn{4}{|c|}{$\begin{array}{l}\text { AsA, L-ascorbic acid; Try, L-tryptophan; Thr, L-threonine; IDY, inac- } \\
\text { tivated dry yeast; CYS, L-cysteine hydrothioride monohydrate; Cys, } \\
\text { L-cysteine p.a.: I lowest amount of individual am ano acid; II, medium } \\
\text { amount of individual amino acid; Ill, highest amount of individual } \\
\text { amino acie. }\end{array}$} \\
\hline
\end{tabular}

(2008). Breadmaking quality was evaluated using the following parameters: tenacity $(P, \mathrm{~mm})$, taken as the height of the curve, which measures the initial resistance to extension; extensibility $(L, \mathrm{~mm})$, taken as the length of the curve; configuration ratio $(P / L)$, which indicates the balance of viscoelastic components; dough swelling (G, $\left.\mathrm{cm}^{3}\right)$; deformation energy $\{W, J)$, which indicates dough strength.

The consistograph parameters evaluated were as follows: PrMax (mbar), maximum pressure; TPrMax (s), time to reach maximum pressure; Tol (s) (tolerance, stability), time when pressure is higher than PrMax minus 20\%; D250 (mbar), drop in pressure at $250 \mathrm{~s}$ from PrMax minus 20\%; D450 (mbar), drop in pressure at $450 \mathrm{~s}$ from PrMax minus $20 \%$.

\section{Baking}

The doughs were prepared from $250 \mathrm{~g}$ flour with $130 \mathrm{gkg}^{-1}$ water, $4.5 \mathrm{~g}$ $\mathrm{kg}^{11}$ salt, $10 \mathrm{gkg}^{-1}$ yeast, $10 \mathrm{gkg} \sim^{1}$ margarine and the corresponding amount of mixture (groups $(-\mathrm{V})$ added. The ingredients were mixed in a spiral stirring machine (HLM-HLM-120, A 1 Kuchyně s.r.o., Plzeň, Czech Republic) for $5.5 \mathrm{~min}$, left to stand for $10 \mathrm{~min}$, kneaded and left to stand for a further $10 \mathrm{~min}$. The doughs were then cut into separate pieces in the shape of a bun using a dough divider (HLM-NFK30, Gastroform s.r.o., Brno, Czech Republic). The pieces were put on a baking tray and left to rise for

$30 \mathrm{~min}$ at a temperature of $35{ }^{\circ} \mathrm{C}$ and a relative humidity of $68 \%$. They were then baked for $10 \mathrm{~min}$ at a temperature of $250{ }^{\circ} \mathrm{C}$.

Sensory analysis

Model bread (in the form of buns) was evaluated by a panel of nine assessors (experts from Ireks Enzyma s.r.o., Brno, Czech Republic) trained according to ISO 8586-2 (1994). The
Table 2. Values of dry matter content in individual mixtures of additives

\begin{tabular}{|c|c|c|c|}
\hline \multirow[b]{2}{*}{ Group } & \multirow[b]{2}{*}{ Mixtures of additives } & \multicolumn{2}{|c|}{ Dry matter content $\left(g \mathrm{~kg}^{-}\right.$; } \\
\hline & & Minimum & Maximum \\
\hline 1 & $A 5 A-T r y|-| l l$ & 397.9 & 402.5 \\
\hline H. & $A S A=T h r l-J I I$ & 397.5 & 407.4 \\
\hline lin & $A_{5} A-|D Y|-|| \mid$ & 396.2 & 399.0 \\
\hline $\mathrm{JV}$ & $A S A+C Y S|-| I$ & 395.4 & 398.4 \\
\hline v & $C y s-\mid\left[D^{y}|-| I \mid\right.$ & 393.1 & 395.9 \\
\hline \multicolumn{4}{|c|}{$\begin{array}{l}\text { "AsA, L-ascorbic acid; Try,L-tryptophan; Thr, L-threonine; IOY, inac- } \\
\text { tivated dry yeast; GYS, L-cysteine hydrochloride monohydrate; Cys, } \\
\text { L-cysteine p.a.i, lowest amount of individual amino acid; Il, medium } \\
\text { amount of individual amino acid; II, highest amount of individual } \\
\text { anino acid. }\end{array}$} \\
\hline
\end{tabular}

\begin{tabular}{|c|c|c|c|}
\hline \multirow[b]{2}{*}{ Group } & \multirow[b]{2}{*}{ Mixtures of additives" } & \multicolumn{2}{|c|}{$\mathrm{pH}$} \\
\hline & & Minimum & Maximustit \\
\hline 1 & ASA $+T T y \mid-\| I I$ & 5.82 & 6.14 \\
\hline \| & $A S A+T h r l-I I I$ & 5.92 & 6.05 \\
\hline III & $A 5 A+|D Y Y-I I|$ & 6.00 & 6.17 \\
\hline $\mathrm{N}$ & $A 5 A \div C Y S \mid-I I I$ & 5.96 & 6.01 \\
\hline v & $C_{y 5}+\left|D_{Y}\right|-\mid I I$ & 5.97 & 6.02 \\
\hline \multicolumn{4}{|c|}{ 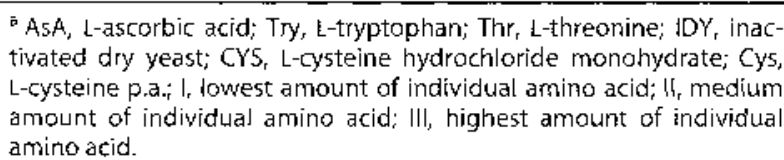 } \\
\hline
\end{tabular}

evaluation was performed in a sensory laboratory equipped in accordance with ISO 8589 (1988). The samples of buns were coded and served at room temperature $\left(22=\mathrm{L} 2{ }^{\circ} \mathrm{C}\right)$. A five- point hedonic scale was used for the assessment of dryness, gumminess, pliability, sensation when swallowing, quality and texture.

Statistical analysis

The results of basic chemical analysis were statistically evaluated. The results of sensory analysis were statistically evaluated by means of non-parametric analysis of variance (Kruskal-Wallis test), Friedman test or Wilcoxon test. ${ }^{35}$ Differences among comparisons had to achieve $P<0.05$ to show significance.

\section{RESULTS AND DISCUSSION}

Chemical analysis

The results obtained by basic chemical analysis showed insignificant differences $(P>0.05)$ in $\mathrm{pH}$ and dry matter content among samples with mixtures of additives (groups (-V), as shown in Tables 2 and 3.

Dry matter content in the control dough (CD) was $393.9 \mathrm{~g} \mathrm{~kg}^{-1}$ and its $\mathrm{pH}$ was 6.00 


\begin{tabular}{|c|c|c|c|c|c|}
\hline \multirow[b]{2}{*}{$\begin{array}{l}\text { Group of mixture } \\
\left(9 \mathrm{~kg}^{-1}\right)\end{array}$} & \multicolumn{5}{|c|}{ Alveographic characteristics" } \\
\hline & $\stackrel{P}{P}$ & $\stackrel{1}{L}$ & $P / 1$ & $\begin{array}{c}G \\
\left(\mathrm{~cm}^{3}\right)\end{array}$ & $\begin{array}{c}W \\
\left(10^{-4} \mathrm{~J}\right)\end{array}$ \\
\hline None (CD) & 95 & 89 & 1.06 & 21.0 & 287 \\
\hline \multicolumn{6}{|c|}{ Group $\mid\left(A_{5 A} A-T r y \mid-\| l\right)$} \\
\hline $1.0+2.0$ & 198 & 48 & 4.15 & 15.4 & 407 \\
\hline $1.0+3.0$ & 200 & 41 & 4.86 & 14.3 & 360 \\
\hline $1.0+4.0$ & 204 & 40 & 5.04 & 14.2 & 359 \\
\hline \multicolumn{6}{|c|}{ Group II (AsA - Thrl-Ill) } \\
\hline $1.0+2.0$ & 198 & 46 & 4.30 & 15.1 & 396 \\
\hline $1.0+3.0$ & 202 & 44 & 4.61 & 14.8 & 388 \\
\hline $1.0+4.0$ & 201 & 47 & 4.32 & 15.2 & 408 \\
\hline \multicolumn{6}{|c|}{ Group III $\{A S A+I D Y \mid-I I\}$} \\
\hline $1.0-2.0$ & 225 & 38 & 5.86 & 13.8 & 382 \\
\hline $1.0-3.0$ & 231 & 38 & 6.14 & 13.7 & 391 \\
\hline $1.0+4.0$ & 212 & 49 & 4.36 & 15.5 & 440 \\
\hline \multicolumn{6}{|c|}{ Group $W\{A S A+C Y \$ 1-1 \mid\}$} \\
\hline $1.0 \div 0.2$ & 114 & 63 & 1.80 & 17.7 & 220 \\
\hline $1.0-0.5$ & 90 & 40 & 2.22 & 14.1 & 110 \\
\hline $1.0 \div 0.6$ & 99 & 23 & 4.26 & 10.7 & 95 \\
\hline \multicolumn{6}{|c|}{ Group V (Cys - IDY|- $\| \mid)$} \\
\hline $0.1+1.0$ & 127 & 61 & 2.09 & 17.4 & 239 \\
\hline $0.1+2.0$ & 137 & 60 & 2.17 & 17.3 & 249 \\
\hline $0.1+3.0$ & 121 & 54 & 2.24 & 16.4 & 210 \\
\hline \multicolumn{6}{|c|}{ 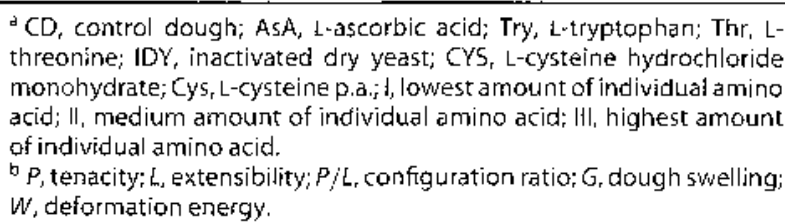 } \\
\hline
\end{tabular}

\section{Rheological analysis}

In order to achieve a complete description of the dough quality needed for baking technology, deformation measurement was used, by means of which the influence of combinations of reducing and oxidising agents on the rheological properties of dough was specified.

Tables 4 and 5 summarise the results for all groups of mixtures added to wheat flour. The same additions of individual groups of mixtures of reducing and oxidising agents were used for dough preparation. If the influence of amino acids and L-ascorbic acid on the rheological properties of dough was not apparent in low amounts, higher amounts were applied.

The individual groups of mixtures (groups $(-\mathrm{V})$ were examined. Thanks to these mixtures, the optimal balance between tenacity, extensibility and stability of the dough and its softening could be reached, which was positively reflected in the quality of the buns.

With increasing addition of L-tryptophan (Tryl-III), group I (AsA + Tryl-III) caused a bigger increase in tenacity but also a bigger decrease in extensibility than group II (Table 4). Furthermore, Table 5 shows that after $250 \mathrm{~s}$ there was also a decrease in stability but a slower softening of the dough. After $450 \mathrm{~s}$ the dough softened in a similar way as it did with the addition of group II.

With increasing addition of L-threonine (Thrl - III), the effect of group II (AsA + Thrl- III) was an increase in tenacity and an uneven decrease in extensibility of the dough (Table 4). Moreover, with increasing addition of this mixture, the dough became less stable
Tabie 5. Consistographic characteristics of doughs investigated

\begin{tabular}{|c|c|c|c|c|c|}
\hline \multirow[b]{2}{*}{$\begin{array}{l}\text { Group of mixture } \\
\left(\mathrm{g} \mathrm{kg}^{-1}\right)\end{array}$} & \multicolumn{5}{|c|}{ Consistographic characteristiss } \\
\hline & $\begin{array}{l}\text { Prilax } \\
\text { (mbar) }\end{array}$ & $\begin{array}{c}\text { TprMax } \\
\text { (5) }\end{array}$ & $\begin{array}{l}\text { Tol } \\
(5)\end{array}$ & $\begin{array}{l}0250 \\
(m b a r)\end{array}$ & $\begin{array}{r}D 450 \\
\text { (mbar) }\end{array}$ \\
\hline None (CD) & 2237 & 173 & 241 & 197 & 742 \\
\hline \multicolumn{6}{|c|}{ Group! (ASA + TryI-III) } \\
\hline $1.0-2.0$ & 2285 & 146 & 304 & 150 & 614 \\
\hline $1.0+3.0$ & 2215 & 178 & 296 & 106 & 540 \\
\hline $1.0+4.0$ & 2101 & 184 & 287 & 95 & 587 \\
\hline \multicolumn{6}{|c|}{ Group il (ASA + Thrl-III) } \\
\hline $1.0+2.0$ & 2306 & 156 & 322 & 190 & 602 \\
\hline $1.0+3.0$ & 2198 & 188 & 326 & 5.1 & 536 \\
\hline $1.0+4.0$ & 2272 & 149 & 302 & 181 & 633 \\
\hline \multicolumn{6}{|c|}{ Group $\||| A S A,+|D Y|-\|||\})$} \\
\hline $1.0+2.0$ & 2150 & 196 & 296 & 61 & 544 \\
\hline $1.0+3.0$ & 2167 & 176 & 317 & 87 & 557 \\
\hline $1.0+4.0$ & 2284 & 206 & 301 & 52 & 528 \\
\hline \multicolumn{6}{|c|}{ Group IV (AsA + CYSi-lli) } \\
\hline $1.0+0.2$ & 2104 & 100 & 114 & 1134 & 1470 \\
\hline $1.0+0.5$ & $229 \pi$ & 90 & 96 & 1665 & 1957 \\
\hline $1.0+0.6$ & 2218 & 81 & 90 & 1723 & 1980 \\
\hline \multicolumn{6}{|c|}{ Group V (Cys + |DY $|-| \mid l)$} \\
\hline $0.1+1.0$ & 2188 & 117 & 144 & 733 & 1048 \\
\hline $0.1-2.0$ & 2172 & 112 & 138 & 799 & 1082 \\
\hline $0.1-3.0$ & 2171 & 113 & 131 & 847 & 1151 \\
\hline
\end{tabular}

${ }^{\mathrm{a}} \mathrm{CD}$, control dough; AsA, L-ascorbic acid; Try, L-tryptophan; Thr, Lthreonine; IDY, inactivated dry yeast; CYS, L-cysteine hydrochloride monohydrate; Cys, L-cysteine p.a.; I, lowest amount of individual amino acid; II, medium amount of individual amino acid; III, highest amount of individual amino acid.

b PrMax, maximum pressure; TPrMax, time to reach maximum pressure; Tol, time when pressure is higher than PrMax minus 20\%; D250, drop in pressure at $250 \mathrm{~s}$ from PrMax minus 20\%; D450, drop in pressure at $450 \mathrm{~s}$ from PrMax minus $20 \%$.

owing to the instability of L-ascorbic acid, as also reflected by the slow but uneven softening of the dough after 250 and $450 \mathrm{~s}$ (Table 5).

It is generally known that oxidising agents are suitable additives for weak flours. Thanks to their ability to strengthen the gluten structure, the dough shows greatertenacity and elasticity. Stronger oxidising additives form a firmer dough structure and improve the cohesiveness of dough, especially when weak flours are used.

With increasing addition of inactivated dryyeast (IDYI-III), group III (AsA + IDYI-III) caused an increase in dough tenacity and also a slow increase in extensibility, which was also higher in comparison with groups I and II (Table 4). Moreover, Table 5 shows that with increasing addition of group III the dough became more stable and, compared with groups I and II, softened more slowly after both 250 and $450 \mathrm{~s}$.

With increasing addition of L-cysteine hydrochloride monohydrate (CYSI-III), group IV (AsA + CYSI-III) caused a decrease in both tenacity and extensibility owing to the reducing effects of L-cysteine hydrochloride monohydrate (Table 4). Our results are in agreement with those of Angioloni and Rosa ${ }^{19}$ and Miller and Hoseney. ${ }^{20}$ Furthermore, there was a decrease in dough stability and its fast softening (Table 5). Our results are in partial agreement with those of Elkhalifa and El-Tinay. ${ }^{21}$ This effect is caused by the ability of cysteine to break disulfide bridges and damage 
Table 6. Results of sensory analysis of tested buns with additive mixtures from groups $1-V$

\begin{tabular}{|c|c|c|c|c|c|c|c|}
\hline \multirow[b]{2}{*}{ Group } & \multirow[b]{2}{*}{$\begin{array}{l}\text { Amount of mixture } \\
\text { added ( } \mathrm{g} \mathrm{kg} \cdot{ }^{*-7} \text { ) }\end{array}$} & \multicolumn{6}{|c|}{ Sensory evaluation (median) $)^{\mathrm{a}, \mathrm{b}}$} \\
\hline & & Dryness & Gumminess & Pliability & $\begin{array}{l}\text { Sensation when } \\
\text { swallowing }\end{array}$ & Quality & Texture \\
\hline \multirow[t]{4}{*}{1} & None & $3 a$ & $4 a$ & $3 a b$ & $3 a$ & $3 a$ & $3 a$ \\
\hline & $1.0+2.0$ & $3 a$ & $3 a$ & $2 z$ & $3 a$ & $2 a$ & $3 a$ \\
\hline & $1.0+3.0$ & 3. & $2 a$ & 3ab & $3 a$ & $3 a$ & $3 a$ \\
\hline & $1.0+4.0$ & $3 . a$ & $3 a$ & $4 b$ & $2 a$ & $2 a$ & $2 a$ \\
\hline \multirow[t]{4}{*}{ ॥ } & None & $2 a$ & $3 \mathrm{a}$ & $4 a$ & $3 a$ & $4 a$ & $4 a$ \\
\hline & $1.0+2.0$ & $3 \mathrm{~b}$ & $3 a$ & $3 b$ & $3 a$ & $3 a$ & $3 a$ \\
\hline & $1.0+3.0$ & $3 a b$ & $3 a$ & $3 a b$ & $3 a$ & $3 a$ & $3 a$ \\
\hline & $1.0+4.0$ & $3 a b$ & $3 a$ & $3 \mathrm{ab}$ & $3 a$ & $4 a$ & $4 a$ \\
\hline \multirow[t]{4}{*}{ III } & None & $2 a$ & $2 a$ & $4 a$ & $4 a$ & $4 a$ & $4 a$ \\
\hline & $1.0+2.0$ & $3 a b$ & $3 a$ & $4 a b$ & $3 a b$ & $3 a b$ & $4 a b$ \\
\hline & $1.0+3.0$ & $3 a b$ & $3 a$ & $2 c b$ & 马七 & $3 b$ & $3 a b$ \\
\hline & $1.0+4.0$ & $4 b$ & $4 a$ & $2 c$ & $2 b$ & $2 b$ & $2 \mathrm{~b}$ \\
\hline \multirow[t]{4}{*}{ IV } & None & $\$ 3$ & $3 a$ & $3 a b$ & $3 a b$ & $3 a b$ & $3 a b$ \\
\hline & $1.0+0.2$ & $3 a$ & $3 a$ & $3 a$ & 20 & 2a & 2a \\
\hline & $1.0+0.5$ & $2 a$ & $3 a$ & $4 a b$ & 4 & $4 a b$ & 4b \\
\hline & $1.0+0.6$ & $2 \mathrm{a}$ & $3 a$ & $4 b$ & $4 b$ & $4 b$ & $4 b$ \\
\hline \multirow[t]{4}{*}{$\mathrm{v}$} & None & $2 a$ & $3 a$ & $4 a$ & $4 a$ & $2 a$ & $3 a$ \\
\hline & $0.1+1.0$ & $3 a b$ & $3 a$ & $4 a$ & $3 a$ & 3 a & $3 a$ \\
\hline & $0.1-2.0$ & $3 a b$ & $3 a$ & $3 a$ & 3. & 4 & 43 \\
\hline & $0.1-3.0$ & $3 \mathrm{~b}$ & $3 a$ & $2 a$ & 3. & $4 a$ & $4 a$ \\
\hline
\end{tabular}

${ }^{a}$ The following hedonic scales were used. Dryness: 1 = very dry to $5=$ very damp. Gumminess: $1=$ very high to $5=$ hardly noticeable. Pliability: $1=$ very high to $5=$ very low. Sensation (when swallowing a bite): $1=$ pleasant to $5=$ bad. Quality: $1=$ excel lent to $5=$ very bad, unacceptable. Texture: $1=$ excellent to 5 = very bad, unacceptable.

${ }^{\mathrm{b}}$ Median values with the same letter in a column are not significantly different $(P>0.05)$; each group was evaluated separately.

the gluten structure, which leads to an increase in pliability and stickiness of the dough, thus making it less cohesive and more difficult to process. L-Cysteine hydrochloride monohydrate is a strong reducing agent and thus should be added to dough in small amount. It is suitable for the preparation of strong flours.

With increasing addition of inactivated dry yeast (IDYI-III), group V (Cys + IDYI-III) caused a slower decrease in tenacity and extensibility of the dough (Table 4). Moreover, there was a slower decrease in dough stability but faster softening, especially after $450 \mathrm{~s}$ (Table 5). Our results are partially consistent with those of Joye eta $1 .^{22}$

\section{Sensory analysis of buns with mixtures from groups I-V}

The results obtained by sensory analysis showed that the gumminess of all buns did not change significantly with increasing amount of mixtures from all groups $(P>0.05)$ (Table 6). Table 6 also shows that dryness decreased in the buns of group II (AsA + Thrl), group III (AsA + IDYIII) and group V (Cys + IDYIII) in comparison with the control (CD) buns. The biggest decrease in dryness occurred in AsA + IDYIII followed by AsA + CYSII compared with AsA + CYSI $(P<0.05)$.

The expert assessors noticed a decrease in pliability of the buns with AsA + Trylll compared with those with AsA + Tryl. On the other hand, an increase in pliability compared with the $C D$ buns was observed in the buns with AsA + Thrl followed by those with AsA + IDYII. Pliability also increased in the buns with AsA + IDYIII compared with those with AsA + IDYI $(P<0.05)$.

A better sensation when swallowing was observed in the buns with AsA + IDYII, III compared with the CD buns. On the other hand, a worse sensation when swallowing occurred in the buns with AsA + CYSII, III compared with those with AsA + CYSI $(P<$ 0.05).

According to the expert assessors, the buns with AsA + IDYII,!!! were of better quality than the CD buns. On the other hand, worse quality was observed in the buns with AsA + CYSIII compared with the CD buns and also in the buns with AsA + CYSII, III compared with those with AsA + CYSI $(P<0.05)$.

Texture improved in the buns with Cys + IDYIII compared with the CD buns. On the other hand, it deteriorated in the buns with AsA + CYSIII compared with the CD buns and also in the buns with AsA + CYSII,III compared with those with AsA + CYSI $(P<0.05)$.

However, no significant differences in dryness, sensation when swallowing, quality, texture and gumminess were observed between the CD buns and the buns with AsA -h Tryl-III. Moreover, no significant differences in dryness and gumminess were found between the $C D$ buns and the buns with AsA + CYSI-III. Also, no significant differences in pliability, sensation when swallowing, quality, texture and gumminess $(P>0.05)$ were found between the $C D$ buns and the buns with Cys + IDYI-111.

\section{CONCLUSIONS}

The effects of the combination of reducing and oxidising agents on the viscoelastic properties of dough and the sensory characteristics of buns were investigated.

Chemical analyses of the dough samples revealed that the $\mathrm{pH}$ did not change with increasing addition of the combination of mixtures to the dough. With respect to the comparison of rheological changes, it cannot be said that the change in $\mathrm{pH}$ 
caused by the addition of mixtures of reducing/oxidising agents influences the binding capacity of the dough. It is solely the influence of the mixtures of reducing/oxidising agents caused by their chemical characteristics that leads either to an increased capability to create hydrogen bridges (oxidation effect) or other weak binding interactions or, on the contrary, to blocking or reducing theformation ofthiol bridges (reducing effect). Moreover, there was no change in dry matter content compared with the control dough. Thus the combination of mixtures did not affect the moistness of the dough either.

According to the rheological results, within groups l-lll (AsA + TrylIII, AsA+Thrl-III and AsA + IDYI-III respectively), group I (AsA + Tryl111) and group III (AsA + IDYI-III) can be recommended thanks to slower softening after 250 and $450 \mathrm{~s}$. Otherwise, the effects of the above-mentioned mixtures are generally comparable and they can be recommended as suitable mixtures of additives to 'weak' flours (flour with damaged gluten). In general, these mixtures are able to strengthen the gluten network and thus increase dough elasticity. Such bakery products show a greater volume and better porosity.

In groups IV and V (AsA + CYSI-111 and Cys + IDYI-III respectively) at the additions given, group $V$ (Cys 4 - IDYI-III) proved to be better thanks to the fact that it provides better tenacity, elasticity and stability of the dough. Unlike groups I-IIl, these mixtures are able to weaken the gluten network and thus increase dough extensibility and decrease its elasticity, which leads to a smooth course of fermentation. They are suitable for flours with 'solid' gluten (flour with quality gluten).

The sensory analysis revealed a significant decrease in dryness in the buns with AsA + Thrl, AsA + IDYIII and Cys + IDYIII compared with the control (CD) buns. The most significant decrease in dryness occurred in the buns with ASA + IDYIII. In comparison with the CD buns, an increase in pliability occurred in the buns with AsA + Thrl and AsA + IDYII. Compared with the CD buns, the sensation when swallowing improved in the buns with AsA + IDYIII. The buns with AsA + IDYII,III showed a better quality than the CD buns. The texture improved in the buns with Cys + IDYIII in comparison with the CD buns.

On the other hand, the buns with AsA + CYSIII showed a significantly worse quality and texture compared with the control CD buns. This combination of additives leads to considerable destruction of the gluten structure due to the effect of L- cysteine hydrochloride monohydrate. Therefore this mixture is more suitable to add to strong flours.

The gumminess of all buns did not change significantly with increasing amount of mixtures.

Also, group I (AsA + Tryl-III) and group II (AsA + Thrl-III) are recommended to be added in small or medium amounts, because large amounts, i.e. AsA + Trylll and AsA + Thrill, have undesirable effects on the gluten structure, as they lead to great strengthening of this structure, which is not suitable in flours with good quality gluten. However, it can be used in flours with poor gluten structure (bad quality gluten), where such strengthening is desirable.

Therefore the combination of instrumental methods and sensory analysis is useful for qualitative evaluation of the final bakery products obtained with mixtures of reducing and oxidising additives.

These mixtures were added to improve the viscoelastic properties of wheat flour dough, which leads to good quality bakery products. Moreover, they are also important from the nutritional point of view, because there are only small amounts of essential amino acids (mainly L-threonine and L-tryptophan) in wheat flour and thus their addition to wheat flour increases the nutritional value of the final bakery product (buns).

\section{ACKNOWLEDGEMENT}

This work was kindly supported by a project of the Czech Ministry of Education, Youth and Sports (grant MSM 7088352101 ).

\section{REFERENCES}

1 Song $Y$ and Zheng $Q$, Dynamic rheological properties of wheat flour dough and proteins. Trends Food Sci Techno! 18:132-138 (2007).

2 MacRitchie $F$, Evaluation of contributions from wheat protein fractions to dough mixing and breadmaking. J Cereal Sci 6:259-268 (1987).

3 MacRitchie F, Physicochemical properties of wheat proteins in relation to functionality. Adv Food NutrRes 36:1 -87 (1992).

4 WeegelsPL, Hamer RJ and Schofield JD, Functional properties of wheat glutenin. J Cereal Sci 23:1 -18 (1996).

5 Jood S, Schofield JD, TsiamiAA and BolleckerS, Effect of glutenin subfractions on bread-making quality of wheat. J Food Sci Technol 36:573-584 (2001).

6 Payne PI, Nightingale MA, Krattiger AF and Holt LM, The relationship between HMW glutenin subunit composition and the breadmaking quality of British-grown wheat varieties. J Sci Food Agric 40:51 -65 (1987).

7 Southan $M$ and MacRitchie $F$, Molecular weight distribution of wheat proteins. Cereal Chem 76:827-836 (1999).

8 Suchy J, Lukow OM and Fu BX, Quantification of monomeric and polymeric wheat proteins and the relationship of protein fractions to wheat quality. J Sci Food Agric 83:1083-1090 (2003).

9 Uthayakumaran S, Gras PW, Stoddard FL and Bekes F, Effect of varying protein content and glutenin to gliadin ratio on the functional properties of wheat dough. Cereal Chem 76:389-394 (1999).

10 Uthayakumaran S and Lukow OM, Functional and multiple end-use characterization of Canadian wheat using a reconstituted dough system. 7 Sci Food Agric 83:889-898 (2003).

11 Uthayakumaran S and Lukow OM, Improving wheat for bread and tortilla production by manipulating glutenin-to-gliadin ratio. J Sci Food Agric 85:2111 - 2118 (2005).

12 Yong-qiang L, Ren-jun $\mathrm{Z}$ and Ji-chun $\mathrm{T}$, Influence of wheat protein contents and fractions on dough rheological properties as determined by using a reconstitution method. China Agric Sci 7:395-404 (2008).

13 Goesaert H, Brijs K, Veraverbeke WS, Courtin CM, Gebruers K and Delcour JA, Wheat flour constituents: how they impact bread quality, and how to impact their functionality. Trends Food Sci Technol 16:1230 (2005).

14 Broeck HC, America AHP, Smulders MJM, Bosch D, Hamer RJ, Gilissen UWJ, etal, A modified extraction protocol enables detection and quantification of celiac disease-related gluten proteins from wheat. JChromatogr B 8:975-982 (2009).

15 TorbicaA, Antov M, MastilovicJ and KnezevicD, The influence of changes in gluten complex structure on technological quality of wheat (Triticum aestivum L.). Food Res Int 40:1038-1045 (2007).

16 Wieser H, Chemistry of gluten proteins. Food Microbiol 24:115-119 (2007).

17 WrigleyCW, Giant proteins with flour power. Nature 381:738-739 (1996).

18 Fitchett CS and Frazier PJ, Action of oxidants and other improvers, in Chemistry and Physics of Baking, ed. by Blanshard JMV, Frazier PJ and GalliardT. Royal Society of Chemistry, London, pp. 179-198 (1986).

19 Angioloni A and Rosa MD, Effects of cysteine and mixing conditions on white/whole dough rheological properties J Food Eng 80:18-23 (2007).

20 Miller KA and HoseneyRC, Effect of oxidation on the dynamic rheological properties of wheat flour-water doughs. Cereal Chem 76:100-104(1999).

21 Elkhalifa AEO and El-Tinay AH, Effect of cysteine on bakery products from wheat-sorghum blends. Food Chem 77:133-137 (2002).

22 Joye IJ, Lagrain B and Delcour JA, Endogenous redox agents and enzymes that affect protein network formation during breadmaking - a review. J Cereal Sci 50:1 -10 (2009). 
23 Dong W and Hoseney RC, Effects of certain breadmaking oxidants and reducing agents on dough rheological properties. Cereal Chem 72:58-64(1995).

24 Hrušková $M$ and Novotná $D$, Effect of ascorbic acid on the rheological properties of wheat fermented dough. Czech J Food Sci 21:137-144 (2003).

25 BloksmaAH and BushukW, Rheology and chemistry of dough, in Wheat Chemistry and Technology, ed. by PomeranzY. American Association of Cereal Chemists, St Paul, MN, pp. 131 - 217 (1988)

26 Agyare KK, Xiong YL, Addo K and Akoh CC, Dynamic rheological and thermal properties of soft wheat flour dough containing structured lipid. JFoodSci 69:297-302 (2004).

27 Dreese PC, Faubion JM and Hoseney RC, Dynamic rheological properties of flour, gluten, and gluten-starch doughs. I. Temperaturedependent changes during heating. Cereal Chem 65:348-353(1988).

28 Singh $\mathrm{H}$ and MacRitchie $\mathrm{F}$, Application of polymer science to properties of gluten. J Cereal Sci 33:231 -243 (2001).

29 Graveland A, Bosveld P, Lichtendonk WJ and Moonen JHE, Superoxide involvement in the reduction of bisulfide bonds of wheat gel proteins. Biochem Biophys Res Commun 93:1189-1195 (1980).
30 Veraverbeke WS, Courtin CM, Verbruggen IM and Delcour JA, Factors governing levels and composition of the sodium dodecyl sulphateunextractable glutenin polymers during straight dough breadmaking. J Cereal Sci 29:129-138 (1999).

31 Jeanjean MF, Damidaux R and Feillet P, Effect of heat treatment on protein solubility and viscoelastic properties of wheat gluten. Cereal Chem 57:325-331 (1980).

32 Lavelli V, Guerrieri N and Cerletti P, Controlled reduction study of modifications induced by gradual heating in gluten proteins .JAgric Food Chem 44:2549-2555 (1996).

33 Schofield JD, Bottomley RC, Timms MF and Booth MR, The effect of heat on wheat gluten and the involvement of sulphydryl-disulphide interchange reactions. J Cereal Sci 1:241 -253 (1983).

34 Weegels PL, Groot AMG, Verhoek JA and Hamer RJ, Effects on gluten of heating at different moisture contents. II. Changes in physicochemical properties and secondary structure. J Cereal Sci 19:39-47 (1994).

35 Agresti A, Analysis of Ordinal Categorical Data. Wiley, New York, NY, p. 287(1984) 